\title{
ULTRASTRUCTURE STUDY OF THE TRANSGENIC REN2 RAT AORTA - PART 2: MEDIA, EXTERNAL ELASTIC LAMINA, AND ADVENTITIA
}

\author{
Melvin R Hayden ${ }^{1-3}$, James R Sowers ${ }^{1-5}$, and Vincent G DeMarco ${ }^{2-5}$ \\ University of Missouri, Columbia School of Medicine, Departments of Internal Medicine ${ }^{1}$, Endocrinology, \\ Diabetes and Metabolism², Diabetes and Cardiovascular Disease Center ${ }^{3}$, Physiology and Pharmacology ${ }^{4}$, and \\ the Harry S Truman VA Medical Center ${ }^{5}$
}

\section{Background}

The renin-angiotensin-aldosterone system (RAAS) plays an important role in the development and progression of vascular stiffness, hypertension and accelerated atherosclerosis, which are associated with the metabolic syndrome (MetS) and type 2 diabetes mellitus. In addition to the intima, RAAS plays an important role in vascular media and adventitial remodeling.

\section{Methods}

Descending thoracic aortas of young male transgenic heterozygous (mRen2) 27 (Ren2) rats were utilized for ultrastructural study. This lean model of hypertension, insulin resistance, and oxidative stress harbors the mouse renin gene and is known to have increased aortic tissue levels of angiotensin II, angiotensin type 1 receptors, and elevated plasma aldosterone levels.

\section{Results}

Ultrastructural observations substantiate known and novel findings in the tunica media, internal and external elastic lamina, and tunica adventitia, which includes: increased media collagen - proteoglycan matrix expansion, increased secretory and proliferative activity and migration of vascular smooth muscle cells (VSMCs) into a newly developing subendothelial neointima, increased VSMC caveolae, mitochondria degeneration, apoptosis; and lipid retention at the elastin lamellar interface. Openings in the external elastic lamina allow pericyte-to-VSMC contacts. The tunica adventitia exhibits stromal pericyte hyperplasia with actively synthetic phenotype and pericyte-pericyte connections.

\section{Conclusion}

While these studies only represent a single snapshot in time, they provide an evaluation of early abnormal ultrastructural vascular remodeling in Ren-2 models of the conduit-elastic thoracic aorta. Biomed Rev 2019;30:111-123

Key words: aldosterone. angiotensin II, extracellular matrix, hypertension, intima, NADPH oxidase, oxidative stress, type 2 diabetes, vascular stiffness.

Received 10 November 2019, revised 26 November 2019, accepted 28 November 2019.

Correspondence to: Melvin R. Hayden, MD, Research Professor, Department of Internal Medicine, Endocrinology, Diabetes and Metabolism, Diabetes and Cardiovascular Disease Research Group, University of Missouri School of Medicine, Columbia, Missouri, Health Sciences Center, MA410, DC043.00, Columbia, Missouri 65212 USA. Tel.: 01573346 3019; E-mail: mrh29pete@gmail.com 


\section{LIST OF ABBREVIATIONS}

\begin{tabular}{|c|c|}
\hline Ang II & $=$ angiotensin II \\
\hline AKT & $=$ serine/threonine kinase Protein kinase $\mathrm{B}$ \\
\hline $\mathrm{AT}_{1} \mathrm{R}$ & $=$ angiotensin type 1 receptor \\
\hline C & $=$ collagen \\
\hline CVD & $=$ cerebrocardiovascular disease \\
\hline $\mathrm{Ea}$ & $=$ arterial elastosis \\
\hline ECM & $=$ extracellular matrix \\
\hline EEL & $=$ external elastic lamina \\
\hline ER & $=$ endoplasmic reticulum \\
\hline HTN & $=$ hypertension \\
\hline IEL & $=$ internal elastic lamina \\
\hline IR & $=$ insulin resistance \\
\hline MMP & $=$ matrix metallopeptidases \\
\hline MetS & $=$ metabolic syndrome \\
\hline MSC & $=$ mesenchymal stem cells \\
\hline MTOC & $=$ microtubule organizing center \\
\hline NADPH Ox & $\begin{array}{l}=\text { nicotinamide adenine dinucleotide phosphate } \\
\text { reduce oxidase }\end{array}$ \\
\hline NFkB & $=$ nuclear factor kappa B \\
\hline OS & $=$ oxidative stress \\
\hline PGC & $=$ proteoglycan \\
\hline Pc & $=$ pericyte \\
\hline PVAT & $=$ perivascular adipose tissue \\
\hline RAAS & $=$ renin-angiotensin-aldosterone system \\
\hline Ren2 & $\begin{aligned}= & (\mathrm{mRen} 2) 27 \text { heterozygous genetic model of lean } \\
& \text { hypertension }\end{aligned}$ \\
\hline R2 & $=\operatorname{Ren} 2$ \\
\hline ROS & $=$ reactive oxygen species \\
\hline SDC & $=$ Sprague Dawley controls \\
\hline SNS & $=$ sympathetic nervous system \\
\hline T2DM & $=$ type 2 diabetes mellitus \\
\hline TEM & $=$ transmission electron microscope \\
\hline VSMCs & $=$ vascular smooth muscle cells \\
\hline VVG & $=$ Verhoeff Van Gieson stain \\
\hline
\end{tabular}

\section{INTRODUCTION}

Under conditions of atherosclerosis, metabolic syndrome (MetS), hypertension (HTN) and type 2 diabetes mellitus (T2DM) vascular remodeling may occur in all four coats (tunicae) of the aorta (intima, media, adventitia and adiposa). Vascular stiffness (1) and accelerated atherosclerosis (atheroscleropathy) are associated with early vascular remodeling that can be appreciated in detail with transmission electron microscopy (TEM). Part 1 of this two-part series described in detail the aortic remodeling that occurs in the endothelial intima and internal elastic lamina layers and introduced some of the early changes of neointimal remodeling (2).

The MetS is a cardiometabolic disorder clustering obesity, HTN, hyperlipidemia, hyperinsulinemia, insulin resistance
(IR), and oxidative stress (OS) in one subject. Therefore, we chose to study the early ultrastructural cellular and extracellular matrix (ECM, briefly, matrix) remodeling of the media, external elastic lamina, and adventitia in descending thoracic aortas from young male transgenic Ren2 rat obtained from the same thoracic aortic tissue that was utilized in Part 1 of this two-part series (2).

The mouse renin gene is overexpressed in Ren2 models which may be characterized by increased plasma levels of aldosterone and increased local aortic tissue levels of angiotensin II (Ang II) and angiotensin type 1 receptors $\left(\mathrm{AT}_{1} \mathrm{R}\right)(2$, 3). In addition to having an activated local renin-angiotensinaldosterone system (RAAS), this model also manifests a heightened activity of the sympathetic nervous system (SNS). Various treatment modalities such as angiotensin converting enzyme inhibition, renin inhibitors, RAAS blockade and amelioration of OS utilizing tempol (a super oxide dismutase and catalase mimetic) have been previously shown to attenuate the functional and ultrastructural maladaptations in many of the involved organs in the Ren2 model $(2,3)$.

Vascular remodeling is a dynamic process, which involves phenotypic change, cellular growth, migration and death in addition to matrix degradation and accumulation. Classically, there are three distinct coats (tunicae) in conduit elastic arteries: tunica intima, tunica media and tunica adventitia (Fig. 1). There is general consensus that endothelial cells are found only in the intima, vascular smooth muscle cells (VSMCs) in the media, and stromal cells in the adventitia. Stromal cells primarily consist of constitutive connective tissue cells: fibrocytesfibroblasts, myofibroblast and pericytes, vascular structures consisting of vasa vasorum arterioles, venules and capillaries (endothelial cells and pericytes) and in some instances, inflammatory cells (macrophages and lymphocytes) $(4,5)$. For background purposes and orientation to the thoracic aorta we have placed a cartoon of the vascular coats of the descending thoracic aorta and light microscopic images to compare the phenotypic changes to the aortic Ren2 as compared to Sprague Dawley controls (SDC) (Fig. 1, 2) (2).

Observational ultrastructural remodeling changes in the media, external elastic lamina (EEL), adventitia and the genesis of the neointima in the male Ren2 rat model are examined. We previously reported increased blood pressure (BP) and arterial stiffness in young male Ren2 rats (6). Our present goal is to introduce the early ultrastructural remodeling changes in the descending thoracic aorta of the young 9-10 week-old male Ren2 rat model of MetS, HTN, OS, and IR as compared to the Sprague Dawley male littermate 


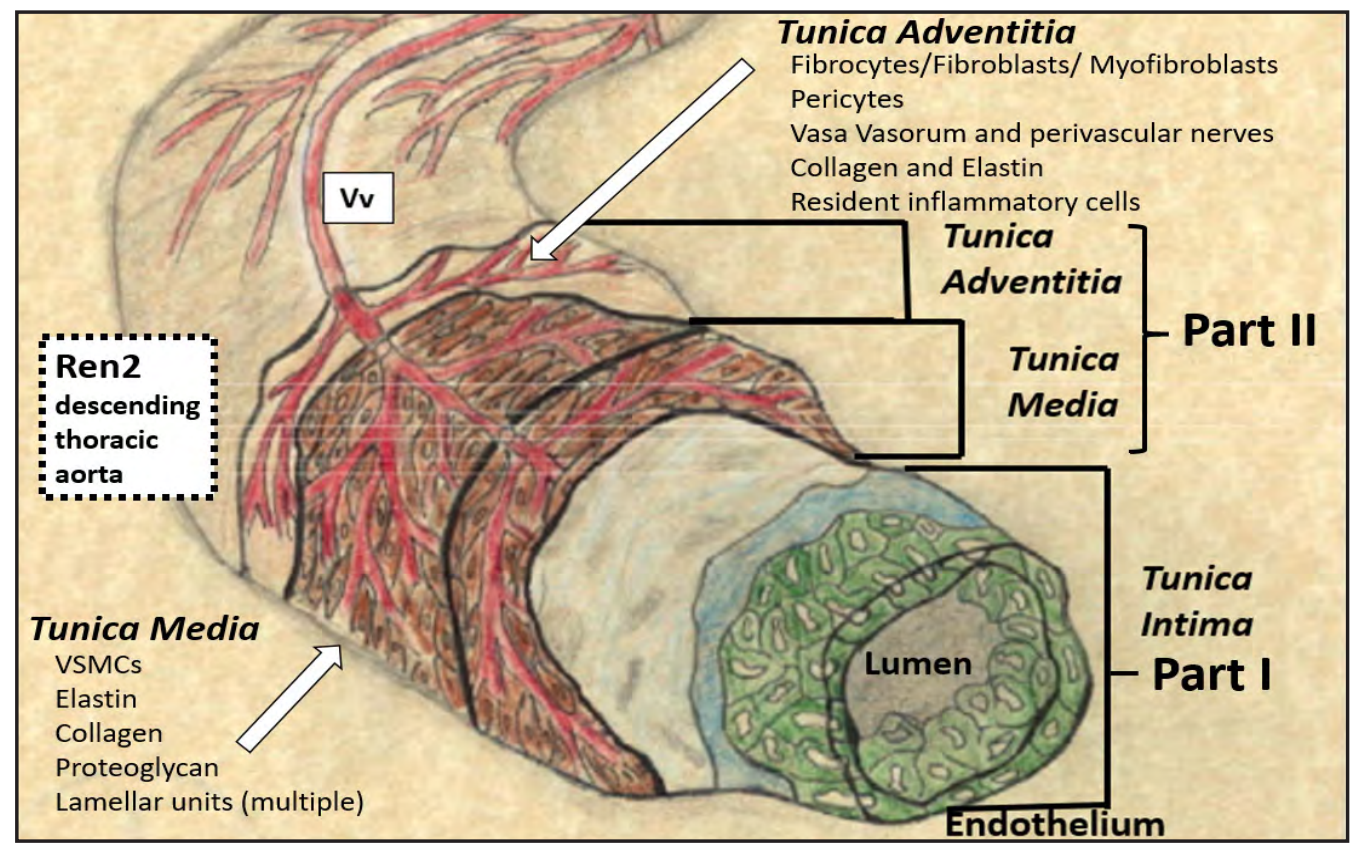

Figure 1. Descending thoracic aorta. This cartoon illustrates each coat of the thoracic aorta: tunica intima, tunica media and tunica adventitia. Tunica adiposa not shown (see 45).

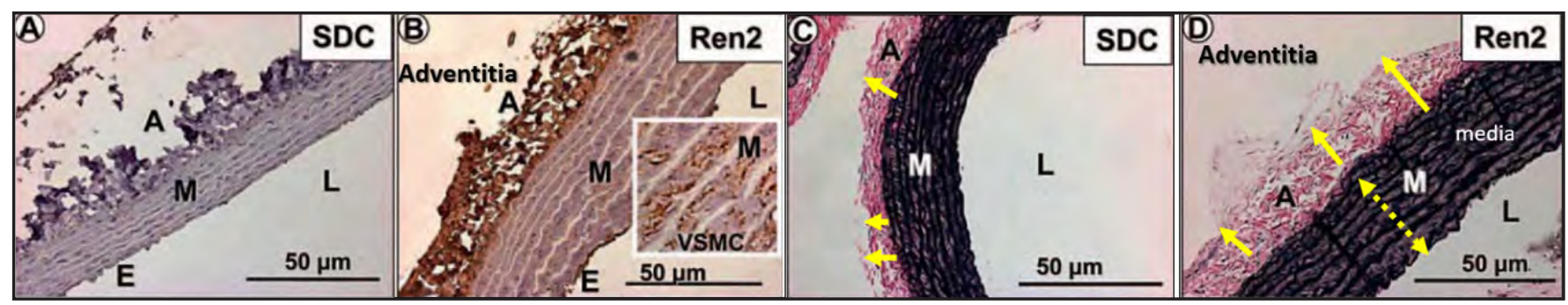

Figure 2. Media and adventitia hypertrophy with increased oxidative stress and expansion collagen viewed by light microscopy. Panel A illustrates the normal cellular phenotype of the tunica media (M) and adventitia (A) in relation to the endothelium (E) of the descending aorta; lumen (L). Panel B_depicts increased oxidative stress in Ren2 models by 3-nitrotyrosine staining. Panel $\boldsymbol{C}$ depicts the normal anatomy of the descending aorta of the media and adventitia in relation to endothelium; elastin stain Verhoeff-Van Gieson (VVG), which also stains collagen I and III pink as in the adventitial layer and interlamerllar regions and adventitia in panels B, C and D respectively. Panel $\mathbf{D}$ demonstrates the hypertrophy of both the media and adventitia (arrows) $a$ with the elastin and collagen stain VVG. Note that both collagen expansion and elastin thickening in the media and the increases collagen pink in the adventitia of the Ren2 models in panels B and D. Scale bar $=50 \mu \mathrm{m}$ in panels A-D. Figure adapted with permission (2).

control model. Understanding early aortic ultrastructural remodeling may allow for a better understanding of the changes in the vasculature during the early development and progression of HTN, vascular stiffness and accelerated atherosclerosis - atheroscleropathy in humans.

\section{MATERIALS AND METHODS}

\section{Animals and Treatments}

Animal care and procedures were approved and followed by the University of Missouri Animal Care and Use Committee and NIH guidelines. Male transgenic (mRen2)27 heterozygous 
$(+/-)$ and age/sex matched SDC littermate rats fed a normal chow diet were sacrificed at 9-10 weeks (2). Blood pressure, weights and glucose were previously described, in which the Ren2 rats were hypertensive, weighed less and had higher fasting blood glucose levels at the time of sacrifice (2). Following harvesting, descending thoracic aortic tissue (upper $1 / 3$ ) was thinly sliced and immediately placed in TEM fixative and prepared for viewing by TEM as previously described (2).

\section{RESULTS}

\section{Media}

The tunica media consists of multiple layers of VSMCs surrounded by a loose areolar collagen and a proteoglycan ECM, which is separated by multiple layers of elastin lamellae; bounded by the internal elastic lamina (IEL) and EEL (Fig.1, 2, 3A). Thoracic aorta is known to be stiffened and thickened in rodent models and human patients with hypertension (1, 5-8). Interestingly, treatment of established HTN does not affect the increase in ECM but decreases VSMC protein content (8). Previous studies of the aorta media in HTN demonstrated increased VSMC synthesis of procollagen/collagen and proteoglycan matrix, which are thought to be primarily responsible for the increase in media volume (8-10). The Ren2 model similarly demonstrated increased organized fibrillar collagen deposition compatible with early fibrosis and proteoglycan deposition (Fig. 3B-D) in the media as compared to SDC.

Vascular SMC were oriented parallel and obliquely to the endothelium and lumen and appeared to have a spider-like appearance due to the multiple cytoplasmic processes extending to the IEL and elastic lamella in both the SDC and Ren2 models (Fig. 3). The SDC and Ren2 VSMCs exhibited cell-cell focal adhesion contacts with desmosomes (Fig. 4A

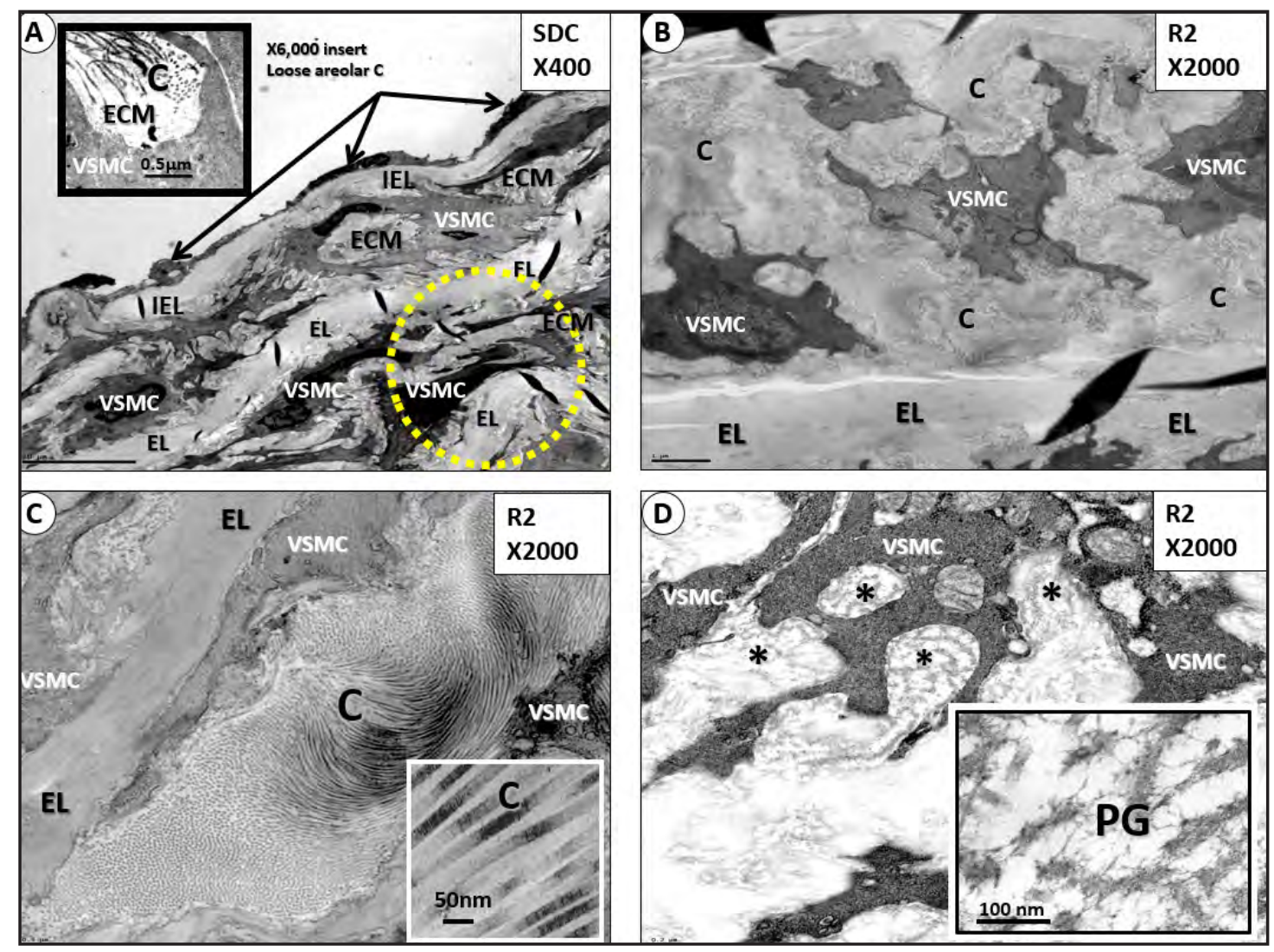

Figure 3. Collagen and vascular smooth muscle cell (VSMC) basal lamina expansion. Panel A illustrates the relationship of the media VSMCs and extracellular matrix (ECM) collagen (C) to the internal elastic lamina (IEL), elastin lamina (EL) and the endothelial cell (EC) monolayer (arrows) in the Sprague Dawley controls (SDC) (magnification x400; bar=10 $\mu \mathrm{m}$ ). Insert depicts loose areolar $C$ in the SDC (magnification x6000; bar $=0.5 \mu \mathrm{m}$ ). Panel $\mathbf{B}-\boldsymbol{D}$ depict excessive accumulation of highly organized fibrillar collagen (C) and multilayered basal lamina (D, asterisks); proteoglycans (?) (PG). Magnification $\times 2,000$; bar = $1 \mu \mathrm{m}$ (panel B); $0.5 \mu \mathrm{m}$ (panel C); $0.2 \mu \mathrm{m}$ (panel D). Insets in panel C and D scale bar $=50 \mathrm{~nm}$ and $100 \mathrm{~nm}$ respectively. 


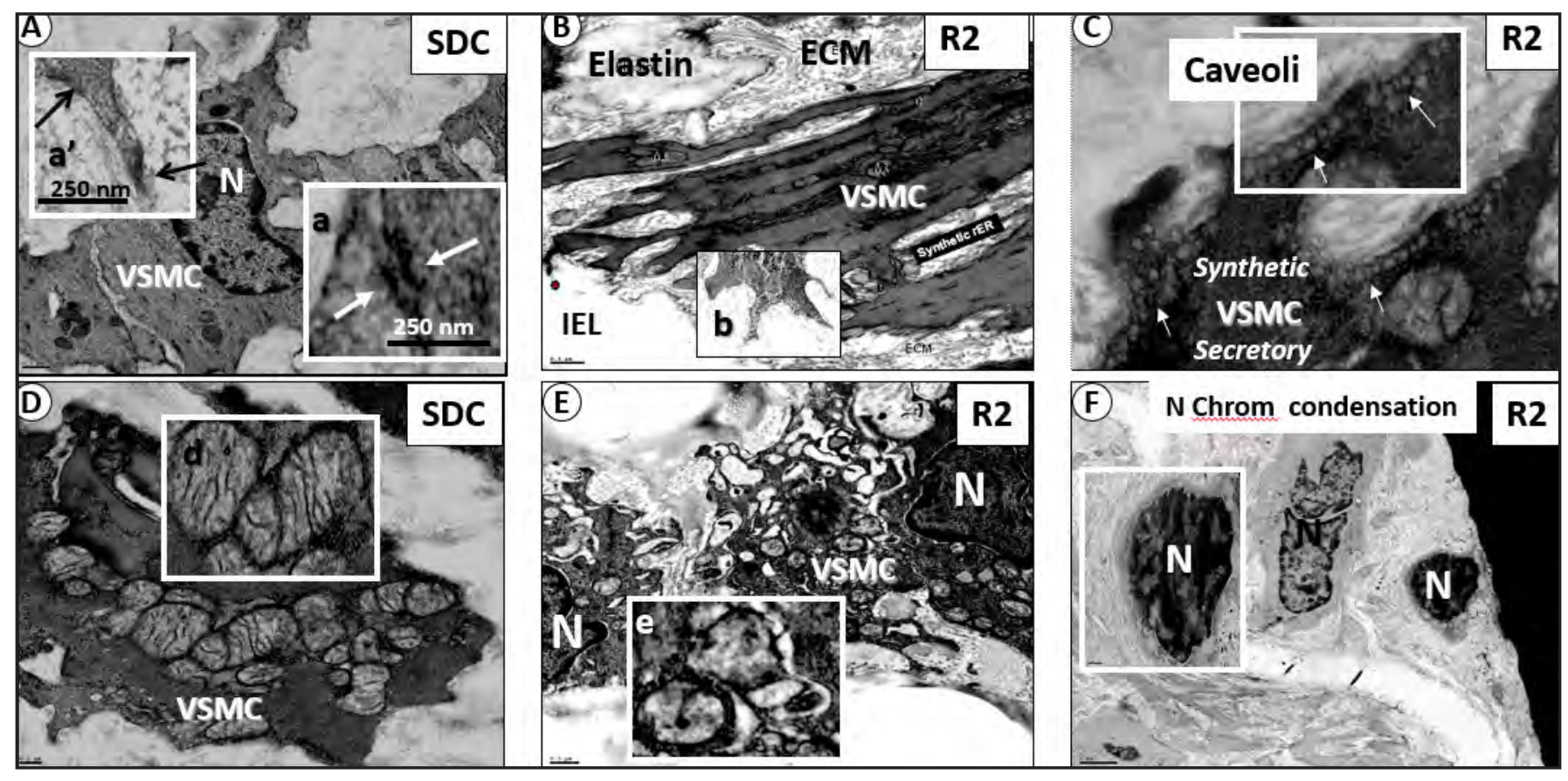

Figure 4. Vascular smooth muscle cell remodeling. Panel A depicts the structure of the cell-cell adherens junction desmosome (arrows) (a) and cell-matrix junction of the hemidesmosomes (arrows) (inserts a and a') in the more electron lucent nonmigratory (contractile phenotype?) vascular smooth muscle cell (VSMC) of the SDC Magnification x2500; bar =0.5 $\mu$ m. Inserts are exploded images of panel A and Bar $=250 \mathrm{~nm} .(N=$ nucleus). Panels $B$ and $\boldsymbol{C}$ demonstrate a synthetic (more electron dense) secretory phenotype (?) of the VSMC the Ren2 (R2) as compared to the SDC in panel A. Note the loss of hemidesmosomes cell matrix junction in insert $\boldsymbol{b}$ as compared to panel $A$ (a and $a$ ' inserts). Also, note the presence of caveolae (arrows) in

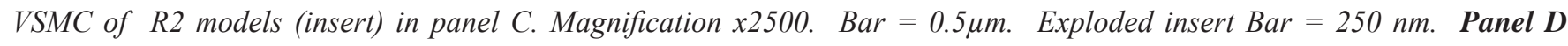
demonstrates normal mitochondria (Mt) with sharply defined cristae in SDC models. Panel $\mathbf{E}$ has less distinct Mt cristae and their matrix reveals a loss in electron density (inset e) indicating maladaptive remodeling and aberrant Mt in R2 models. Note the overall marked increase in R2 cytoplasmic electron density in panels B-E as compared to the SCD in panel A. Panel $\boldsymbol{F}$ depicts "preapoptotic" nuclear chromatin condensation in VSMC in the R2 models. Magnification x 2500; bar =0.2 4 m.

insert a) as previously described $(11,12)$.

The Ren2 vasculature displayed an increase in the secretory VSMC phenotype, which had lost both their cell-cell adherens junction desmosomes) and cell-matrix (hemidesmosome) connections, compatible with an increased secretory-proliferative and migratory activity (Fig. 4B). Ren2 VSMCs also had increased numbers of caveolae at their plasmalemmal surface (Fig. 4C), which may be due to the underlying increased OS similar to the increased secretory vacuoles and caveolae noted in EC (2). Interestingly, there were VSMC remodeling changes of degenerative mitochondria, which consisted of swelling, breaks and loss of cristae and decreased mitochondrial matrix electron density (Fig. 4E) as compared to SDC (Fig. 4D). Further, the presence of nuclear chromatin condensation - clump- ing, nuclear retraction and cytoplasmic apoptotic bodies in VSMCs suggested an apoptotic milieu in Ren 2 vasculature (Fig. 4F). A previous study demonstrated increased apoptosis in the Ren2 thoracic aorta at 9-10 weeks of age and related it to impaired phosphorylation of protein kinase B/AKT (3). These investigators were able to demonstrate an increase in caspase-3 and TUNEL staining in Ren2 vasculature. Additionally, they were able to demonstrate mitochondrial leakage of cytochrome $c$ into the cytosol of the VSMCs. Importantly, there was evidence of lipid deposition in the media at the elastin lamella-VSMC layer interface in the Ren2, which was not observed in the SDC model (Fig. 5B insert). These findings demonstrate that the aorta media layer in the Ren2 vasculature retains lipids, even though there was no evidence of lipid 
retention within the newly created intima at this young age. It is of interest that this region is also the location of calcium deposition; however, in this young model there was no evidence of vascular calcification by TEM studies. Interestingly, there was variable electron dense staining of VSMCs in Ren2 models (Fig. 5A). These findings support the notion that there is increased proliferative and secretory phenotype and migratory activity may be possible in these electron dense VSMCs.

\section{Genesis of the Neointima in the Ren2 Aorta}

Rat vasculature does not have an identifiable subendothelial space (intima) between 6 and 10 weeks and usually does not develop an intima until approximately one year of age (13). This knowledge along with ultrastructural observations allowed for the identification of the genesis of the neointima in the Ren2 model due to metabolic stressors of increased tissue levels of Ang II, OS, IR, increased plasma levels of aldoster-

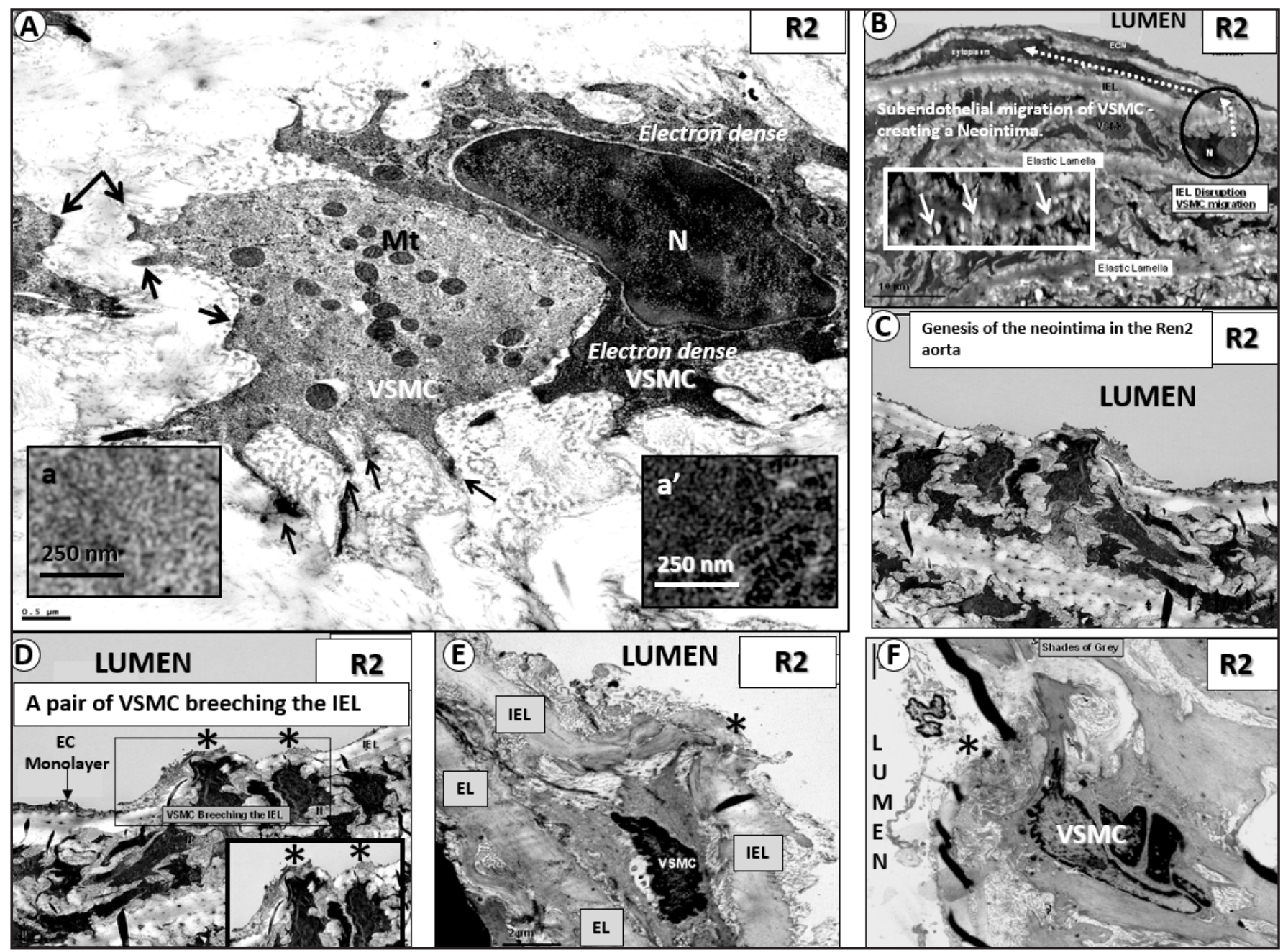

Figure 5. Smooth muscle cell remodeling. Panel A demonstrates two side by side vascular smooth muscle cells (VSMCs). The VSMC on the right illustrates nuclear retraction and has a marked increase in cytoplasmic electron density as compared to the non-migratory quiescent VSMC on the left. Exploded inserts ( $a$ and $a^{\prime}$ ) (bar $=250 \mathrm{~nm}$ ) below each image depicts the difference between electron densities and insert a' illustrates that this electron density is a result of increased synthetic activity (?). as compared to the non-secretory (contractile) phenotype in insert (a). Magnification x2500; bar $=0.5 \mu m$. Importantly, note the electron dense tips (hemidesmosomes) at the tips of the cytoplasmic processes in the left-hand non-synthetic non-migratory VSMCs and that the secretory VSMCs (electron dense) on right hand side appears to not have hemidesmosomes. Panel $\boldsymbol{B}$ depicts the migration of VSMCs into the neointimal region. Magnification x200; bar = 1 $\mu$ m. Panels C-F depict various images and magnifications and scale bar labeled of the migratory - synthetic electron dense VSMCs as they migrate and create the genesis of neointima - subendothelial regions of the aorta.

Biomed Rev 30, 2019 
one, dysglycemia and associated hemodynamic-hypertensive stressors (Fig. 5, 6). Incidentally, the genesis of the neointima in humans develops neonatally, then regresses and progressively develops through adolescence into the adult years (1416). Importantly, it is the intimal structural space between the endothelial monolayer and the IEL where lipids and inflammatory cells are initially deposited allowing the genesis of the fatty streak, which progresses to the fibroatheroma following the migration and expansion of VSMCs within the in- tima. As oxidized lipids continue to accumulate the atheroma becomes more lipid laden with progressive inflammatory changes and eventually undergoes vascular calcifications in association with VSMCs remodeling and apoptosis. It is not feasible to discuss the in-depth development of the unstable plaque, which may result in plaque rupture and thrombosis. However, macrophage inflammation at the shoulders of the plaque and VSMC apoptosis are strongly associated with this type of vulnerable plaque in the complicated process of ath-

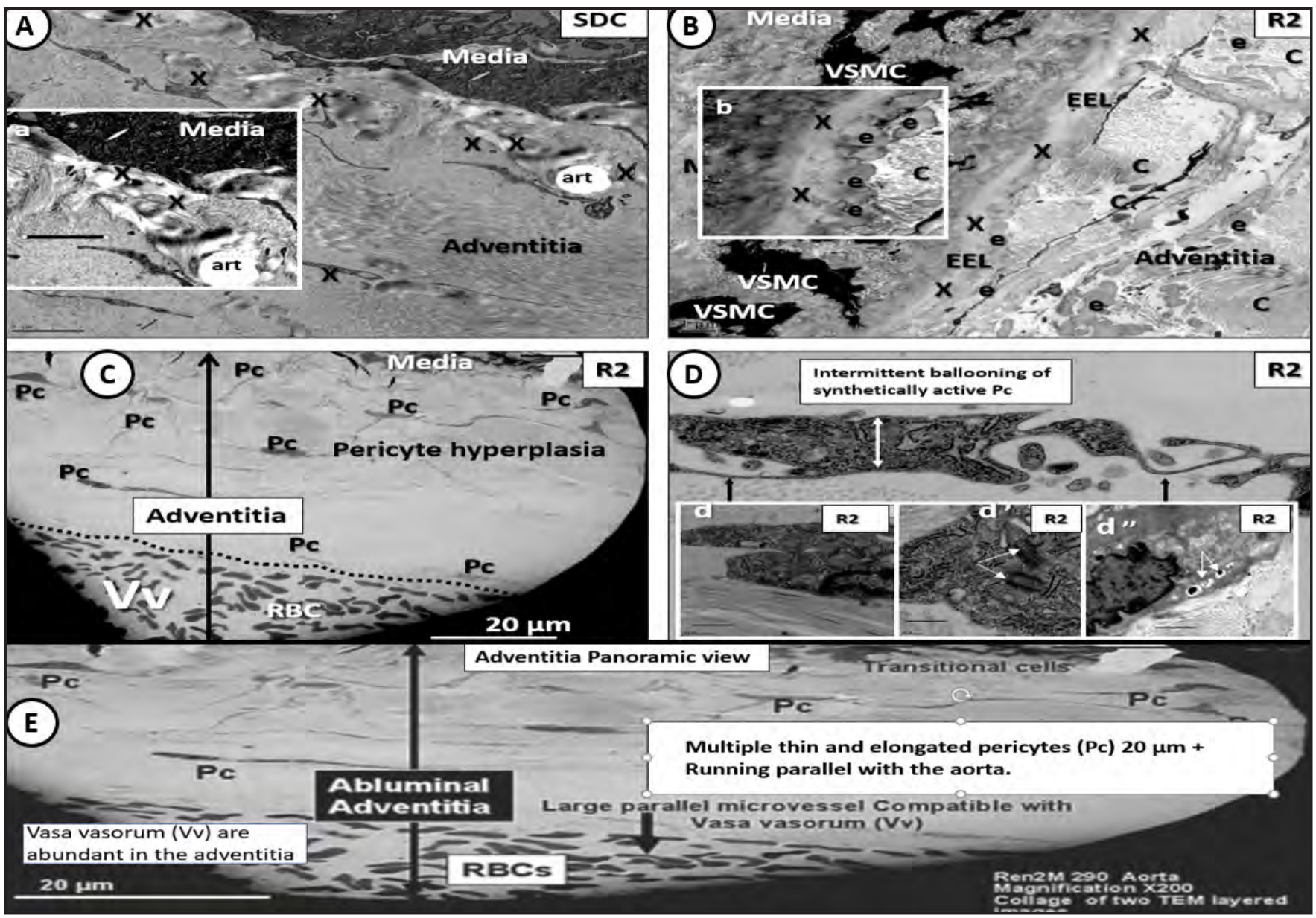

Figure 6. External elastic lamina and adventitia. Panel A illustrates the refractile and interrupted appearance of the external

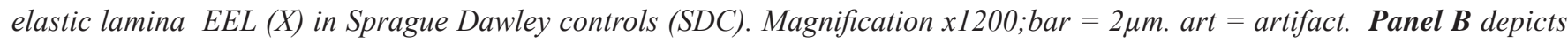
a more homogeneous and less interrupted ELL in Ren2 models (R2). Importantly, note the additional layering of elastin (e) in the Ren2 (R2) models in inset b. Vascular smooth muscle cell(s) (VSMC) are labeled in the media. Magnification x1200; bar = $1 \mu \mathrm{m}$. Insert $b$ is an exploded image of B. $C=$ collagen. Panel $C$ demonstrates pericyte (Pc) hyperplasia within the adventitia. Also note the prominent vasa vasorum (Vv) venule with red blood cell(s) (RBC) in the lumen. Magnification x200; bar = 20 $\mu m$. Panel $\boldsymbol{D}$ depicts a synthetically active Pc with ballooning (double arrows) of thinned segments (arrows) in the hyperplastic region (magnification $x 8000 ;$ bar $=1 \mu \mathrm{m}$ ) and inserts illustrates Pc cell-cell contact (d), (d'and apoptotic bodies (arrows) (d'). Panel $\mathbf{E}$ depicts a more panoramic view of the adventitia where the elongation of pericyte(s) (Pc) exceed in some instances 20 micrometers. $R B C s=$ red blood cells. Varying scale bars are shown in each image. Magnification x8000; bar $=500 \mathrm{~nm}$. 
erosclerosis (16-19).

Ren2 aortic vasculature demonstrated the genesis of a neointima at 9-10 weeks of age in some regions of the aorta and this finding was not present in the SDC model. The creation of a neointima is dependent on the activation of a more synthetic-proliferative VSMC phenotype and its effects on the IEL with an eventual breeching of the IEL with subsequent migration into the neointimal subendothelial space (2, 3 ). There were no findings of lipid deposition in the newly created intima in the young lean hypertenisive Ren2 model; however, this anatomical space is just undergoing its creation. Interestingly, early ultrastructural changes of lipid deposition (verified by oil-red O staining) within the media of the Ren2 was located at the interface of the elastin lamella and VSMC layers (3).

\section{External Elastic Lamina (EEL)}

While some feel that the EEL may serve as a barrier for cells and macromolecules between the adventitia and medial layers of the aortic wall (18), our current observations demonstrate that it is less well developed and found to have more frequent normal interruptions in contrast to the subendothelial IEL and the elastin lamella that separate each layer of the VSMCs of the media in the SDC models in this young Ren2 model of lean HTN (Fig. 6). The EEL acts as a constraining outer elastic membrane of the abluminal portion of the contracting media and is a natural occurring anatomical region, which allows the media and abluminal adventitial regions to be distinguished from one another (18). Ren2 EEL appeared to be more homogeneous, refractile and more continuous; however, it did contain interruptions, which allowed for communication between the adventitia and media (Fig. 6). The medial VSMCs and the abluminal adventitial pericytes-fibrocytes are responsible for the synthesis of elastin in the EEL. Synthesis and layering-on or thickening of adventitial elastin to the existing EEL appeared to be primarily attributable to the abluminal pericytes (Fig. 6B insert b). The EEL appeared to be thickened as compared with the SDC models in this experiment and the EEL is known to undergo remodeling thickening in hypercholesterolemia and hypertension (19, 20). Interestingly, aberrant elastin deposition may play an important role in the development of hypertension (21). As researchers and clinicians, we should be attentive to the evolving story of elastin not only in the media, but also in the adventitial regional remodeling in the development of vascular stiffness, hypertension and atherosclerosis.

\section{Adventitia}

The adventitial layer contains collagen, elastin fibers, fibrocytes, fibroblasts, myofibroblasts and pericyte cells with long cytoplasmic processes, nerve endings and the microvascular vasa vasorum (Fig. 7C, E). The importance of this region in vascular remodeling is increasing and may be a future frontier concerning the developmental remodeling and progression of vascular stiffness, HTN and atherosclerosis. With light microscopy this layer appears to contain mostly types I and III collagen and appears for the most part to be inert (Fig. 2). However, recent information has demonstrated multiple new findings in this layer indicating that this layer may be more than just a supportive extracellular matrix encapsulation of the arterial vessel wall media. These findings include the roles of adventitial pericyte - fibrocytes, fibroblasts- myofibroblasts, inflammation, oxidative stress, matrix expansion and increased vasa vasorum microvessels (angiogenesis) during the development and progression of vascular remodeling in hypertension and accelerated atherosclerosis (22-25).

The adventitial ECM is highly organized at the immediate abluminal EEL regions (compact type I fibrillar collagen) as compared to the loose areolar-type collagen (type III collagen) toward the outermost abluminal regions of adventitia. Also, adventitial collagen fibers were noted to run parallel with the EEL as well as in whorls of highly organized collagen in Ren2 models (Fig. 7B). This region also contained an increase in non- organized regions of elastin protein deposition unrelated to the EEL in Ren2 (Fig. 7B insert 7b). The adventitia was definitely more highly organized on ultrastructural examination than it would appear on light microscopy. Recently, the perivascular adipose tissue (PVAT, tunica adiposa) has become an important player in the pathogenesis of obesity and related cardiometabolic diseases (reviewed in 26, 45); however, the Ren2 is a lean model of hypertension and therefore the contingent PVAT was not purposely collected at sacrifice nor was it intentionally stripped.

The primary cell type in the adventitial layer are the stromal pericytes - fibrocyte, fibroblasts, which demonstrated long cytoplasmic processes up to 20 micrometers or longer (Fig. 6C). In addition to synthesizing predominately collagen, pericytes are also capable of synthesizing elastin in the adventitia and appear to support the VSMC of the media in the maintenance of the EEL. Importantly, pericyte hyperplasia was noted in Ren2 (Fig. 6C) and has been previously documented in rodent models with hypertension $(21,27)$.

The Ren2 aortic adventitial layer demonstrated increased 

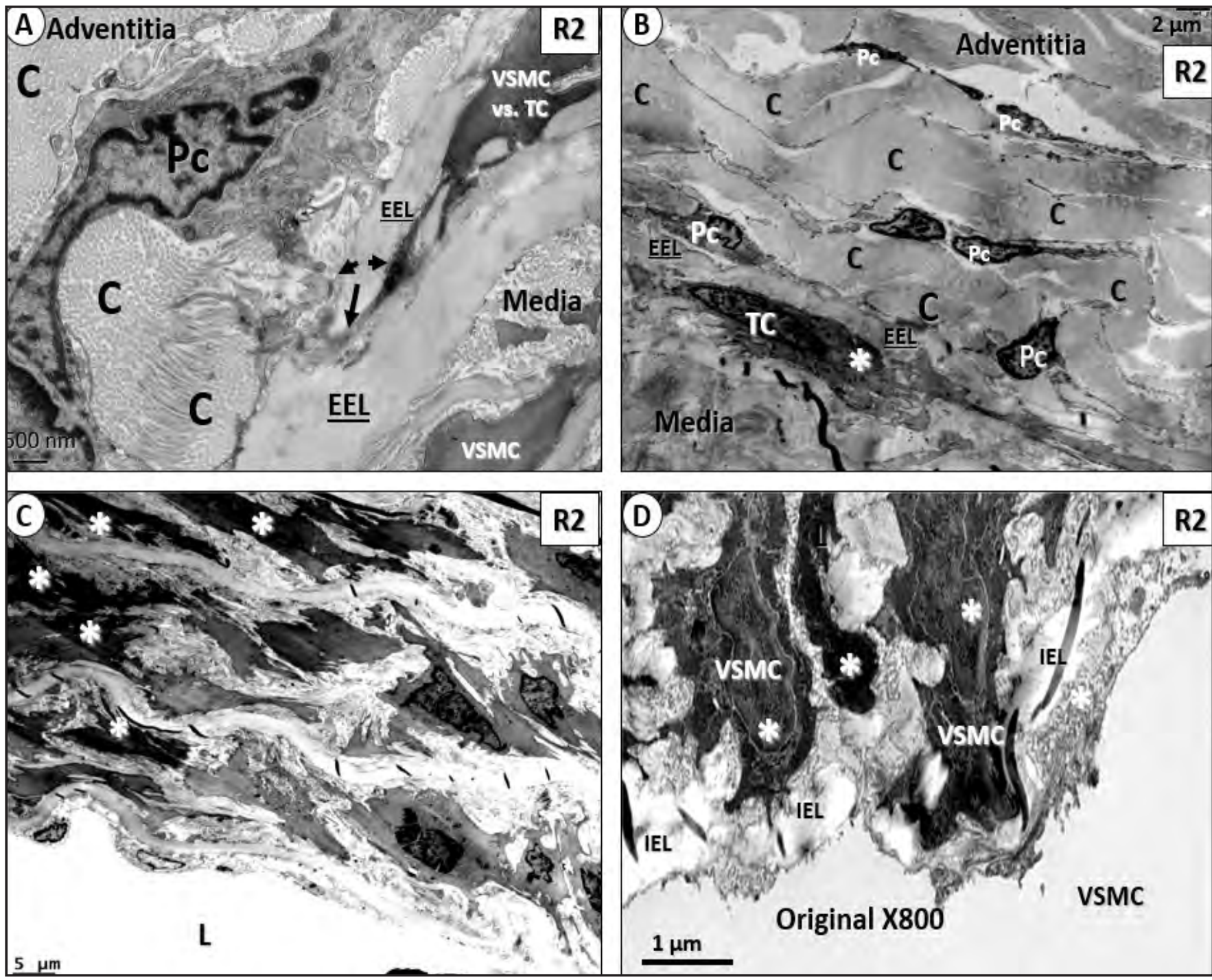

Figure 7. Pericyte-vascular smooth muscle interaction. This collection of images (panels A-D) depict pericyte(s) (Pc), vascular smooth muscle cell (VSMC) interaction at the interface of external elastic lamina (EEL) (arrows) and adventitia (panel A), possible transitional cell (TC) between Pc and VSMC (panel B), electron dense VSMCs representing the pro-synthetic and prosecretory migratory phenotype (asterisks) breeching the internal elastic lamina (IEL) and thus, create the subintimal space in Ren2 models (panels $C$ and D). Note in this image that one can follow the pericyte to the transitional cell (VSMC vs Transitional cell (TC) across the media to the intimal invasion of the internal elastic lamina (IEL), which are identified with asterisks. Varying magnifications with scale bars are included.

OS (found to be primarily due to increased activity of NADPH oxidase), expansion remodeling with widening and pericyte hyperplasia as compared to the SDC (Fig. 2B , 7) $(1,2)$. This hyperplasia was similar to what our group found in the islet exocrine interface of pancreata previously studied in the Ren2 pancreas as well as the human islet amyloid polypeptide (HIP) rat model of T2DM and the $\mathrm{db} / \mathrm{db}$ mouse model of T2DM (28-30). Adventitial pericytes also appeared to be synthetically active with markedly increased electron density associated with abundant endoplasmic reticulum (Fig. 6D) with cytoplasmic enlargements or ballooning as compared to the SDC. In regions of the adventitia - EEL interface of the Ren2 there were frequent pericyte-to-pericyte contact and pericyte -VSMC contacts. Some cells were mor- 
phologically difficult to determine if they were VSMCs or adventitial pericytes at the EEL-adventitial interface, which suggested the possibility of a transitional cell phenotype (pericytes transitioning to VSMCs) (Fig. 7A, B). This notion is feasible, since it is known that pericytes are capable of differentiating into multiple other cell types including VSMCs (30-35). Apoptotic pericytes in the adventitia (Fig. 7C) were noted and adventitial apoptosis in the Ren2 with TdT-mediated dUTP Nick-End Labeling (TUNEL) staining has been previously demonstrated (2).

\section{Specialized Role for Adventitial Pericytes as Adult Mesenchymal Cells}

Mesenchymal stem cells (MSC) are known to reside in postnatal organs (31). Additionally, post-natal undifferentiated pericytes are known to be capable of differentiation into multiple cell types (32-36).

A continuous adventitial - subendothelial - intimal network formed by pericyte-like cells in the adventitia and their processes crossing EEL - media and entering the intima in aortas of humans utilizing anti-pericyte antibody 3G5 staining has been described (37). These findings along with our ultrastructural findings of pericyte-VSMC communication and possible transitional cell forms support the hypothesis that adventitial pericytes may differentiate into VSMC and/or myofibroblasts at the adventitial - EEL interface and migrate across the media to arrive in the newly created intimal space in the Ren2. Ultrastructural observations of adventitial pericytes may allow for a slightly different or co-existing mechanism to supplement our current concepts regarding the role of the myofibroblast and pericyte in the early remodeling changes of atherosclerosis and vascular stiffness in HTN.

\section{DISCUSSION}

The ultrastructural cellular and matrix remodeling occurring within the Ren2 descending thoracic aorta has allowed a better ultrastructural understanding of the early remodeling changes within the arterial vessel wall of the aorta in younger lean hypertensive Ren2 models. Each layer of the aorta in this ultrastructural study has shed new light on the early remodeling changes associated with vascular stiffness, hypertension and the accelerated atherosclerosis - atheroscleropathy associated with HTN, Met S, dysglycemia, excess aortic tissue levels of Ang II and increased $\mathrm{AT}_{1} \mathrm{R}$, hyperaldostronemia and oxidative stress. In addition to the ultrastructural remodeling changes in the endothelium and the internal elastic lamina
(2), there were contemporaneous remodeling changes in the media, genesis of a neointima, expansion of the media ECM and proteoglycans, EEL and adventitia. The loss of cell-cell and cell-matrix connections between the VSMCs may result in apoptosis, which is known to be increased in the Ren2 (3). Thus, the potential for vascular cell apoptosis in all three constitutive vascular aortic cells (endothelium, VSMCs; pericytes) exists.

With endothelial cell activation, apoptosis, denudation and superficial erosion of this monolayer, leukocyte, platelet adhesion and erythrocyte adhesion to the EC and exposed subendothelial layer could develop. This endothelial cell activation could create the potential for the endothelium to increase synthesis of adhesion molecules - chemokines and attract inflammatory monocytes with subsequent absorption and differentiation into activated macrophages within the newly created intimal space due to the invasion of migratory VSMCs or pericyte-derived VSMCs into the newly created neointimal space. Concurrently, endothelial activation would allow for increased lipid absorption into the newly created intima (neointima) and instigate the initiation of the fatty streak, which over time could progress to fibroatheroma and eventually to instability, endothelial superficial erosion with plaque vulnerability and/or plaque rupture and thrombosis, which would result in acute coronary syndromes.

Observations in the media of the Ren2 allowed a morphological ultrastructural differential electron staining of VSMCs. Electron dense secretory phenotype VSMCs as observed and described in the present study (cf 44) demonstrated a loss of cell-cell (desmosomes) and cell-matrix (hemidesmosomes) and focal adhesion connections, which might allow them to readily migrate through the aortic IEL. Importantly, this study demonstrated VSMC migration and breeching of the IEL to create the newly formed subendothelial space (genesis of the neointima) as well as the currently known cause for media enlargement, namely collagen and proteoglycan expansion. The EEL shed light on its importance, in that, the EEL- adventitial interface allowed for increased pericyte-to-VSMC contacts and the possibility to develop transitional phenotypes of the pericyte and VSMC.

Observations of the adventitia revealed an important role of pericyte hyperplasia in the Ren2 with the associated thickening of the adventitia with increased adventitial fibrosis and revealed novel findings regarding the role of its intimate contacts with each constituent adventitial cells as well as the VSMC of the outer media at the EEL - adventitial interface. 
This investigation highlighted what is currently known regarding the early changes of aortic remodeling and has provided novel findings regarding some of the early remodeling changes within the Ren2 aorta. The dynamic interactions of increased vascular tissue levels of Ang II, numbers of $\mathrm{AT}_{1} \mathrm{R}$, hyperaldosteronism, endoplasmic reticulum and oxidative stress are thought to result in the increased production of ROS.

In conclusion, this ultrastructural investigation of the Ren2 aorta has allowed for the collection and study of indelible images involving all the constitutive vascular cell types and ECM in relation to excess local-tissue Ang II, $\mathrm{AT}_{1} \mathrm{R}$, ROS, OS, IR, dysglycemia, hyperaldosteronemia and HTN in the MetS.

\section{FUTURE DIRECTIONS}

Vascular stiffness characterized by increased aortic pulse wave velocity is now considered to be an independent risk factor for increased cardiovascular morbidity and mortality (38). We did not measure the pulse wave velocity in the Ren2 rat in this experiment; however, it is known that HTN induces aortic stiffness and many of the ultrastructural remodeling changes that were observed to be present. We and others have reported on the underlying mechanisms contributing to development of vascular stiffness in diet-induced obesity $(39,40)$.

While we have described ultrastructural remodeling in lean rats in the setting of MetS and lean hypertension, we wish to compare and contrast the ultrastructural remodeling observed herein to those that occur in obese and diabetic models with various treatment modalities such as in the $d b / d b$ mouse or other diabetic models. We hypothesize that descending aortic ultrastructural remodeling that will be associated with vascular stiffness and increased pulse wave velocity with some similarities to the lean hypertensive Ren2 model. Therefore, it is important to observe and understand the ultrastructural remodeling in a lean-controls (SDC) and hypertensive (Ren2) models to better understand and compare the aortic remodeling in future experiments in the obese, insulin resistant, type 2 diabetic models such as the $d b / d b$ models.

The ultrastructural counterpart of the physiologic parameters of the descending aorta is the lamellar unit, which consists of two elastic lamellae encompassing the VSMCs and the ECM of the aortic media $(41,42)$. Interestingly, the C57BL/6 mouse, Ren 2 rat models and humans have five, eight and 50 lamellar units respectively in the descending conduit elastic aorta (43). There is considerable disparity between the number of lamellar units in rodent models and humans; however, it is thought that the ultrastructural remodeling within each lamellar unit will provide ample evidence to notice measurable differences between control, diseased, and treated models, which are necessary in order to sort out some of the differences between lean hypertensive and non-hypertensive obese models.

We have been able to demonstrate that each layer of the descending aorta is extremely important in maintaining the integrity of the vascular circulation function and that each layer may have an early response to various injuries as in the Ren2 lean hypertensive models. Additionally, we are looking forward to better understand the role of aortic stiffness and its association with the rapidly expanding knowledge regarding PVAT (tunica adiposa) in obesity and related diseases $(26,45)$, and may become one of the final frontiers of a more complete understanding of aortic structure and function and aberrant remolding $(46,47)$.

\section{AUTHORS CONTRIBUTIONS}

MRH, VGD, and JRS conceived the idea to instigate this project. MRH obtained and evaluated TEM images. MRH and VGD each played an equal role in writing and editing and JRS provided essential editing.

\section{ACKNOWLEDGEMENT}

This research was supported by NIH (R01 HL73101-01A1) and the Veterans Affairs Merit System (0018), for James R Sowers, M.D. The authors would like to acknowledge the Electron Microscopic Core Center at the University of Missouri, Columbia, Missouri for sample preparation and assistance. Male transgenic Ren2 rats and male Sprague-Dawley controls were kindly provided by the Transgenic Core Facility at the Wake Forest University School of Medicine, Winston-Salem, North Carolina (supported in part by NIH grant HL-51952 to Carlos M. Ferrario)

\section{COMPETING INTERESTS}

The authors declare that they have no competing interests.

\section{REFERENCES}

1. Jia G, Aroor AR, Sowers JR: Arterial stiffness. A Nexus between Cardiac and Renal Disease. Cardiorenal Med 2014;4(1):60-71. doi: 10.1159/000360867

2. Hayden MR, Habibi J, Joginpally T, Karuparthi PR, Sowers JR. Ultrastructure Study of Transgenic Ren2 Rat Aorta - Part 1: Endothelium and Intima. Cardiorenal Med 2012;2(1):66-82. doi:10.1159/000335565 
3. Wei Y, Whaley-Connell AT, Habibi J, Rehmer J, Rehmer $\mathrm{N}$, Patel K, et al. Mineralocorticoid receptor antagonism attenuates vascular apoptosis and injury via rescuing protein kinase B activation. Hypertension 2009;53(2):158165. DOI:10.1161/HYPERTENSIONAHA.108.121954

4. Glagov S. Hemodynamic risk factors: mechanical stress, mural architecture, media nutrition, and the vulnerability of arteries to atherosclerosis. In: The Pathology of Atherosclerosis, Edited by Wissler RW, Geer JC, Kaufman N. Baltimore: Williams and Wilkins; 1972:164.

5. Geer, JC, Haust MD. Smooth muscle cells in atherosclerosis. In: Monographs on Atherosclerosis, Edited by Pollak OJ, Simms HS, Kirk JE. Basal, Karger; 1972: 39.

6. Johnson MS, DeMarco VG, Heesch CM, Whaley-Connell AT, Schneider RI, Rehmer NT, et al. Sex differences in baroreflex sensitivity, heart rate variability, and end organ damage in the TGR(mRen2)27 rat. Am J Physiol Heart Circ Physiol 2011;301: H1540-H1550. doi: 10.1152/ ajpheart.00593.2011

7. Wolinsky H. Effects of hypertension and its reversal on the thoracic aorta of male and female rats. Morphological and chemical studies. Circ Res 1971;28(6):622-637. doi:10.1161/01.res.28.6.622

8. Wiener J, Loud AV, Giacomelli F, Anversa P. Morphometric analysis of hypertension-induced hypertrophy of rat thoracic aorta. Am J Pathol 1977; 88(3):619-634. PMCID:PMC2032372

9. Amann K, Wolf B, Nichols C, Törnig J, Schwarz U, Zeier M, Mall G, Ritz E. Aortic changes in experimental renal failure: hyperplasia or hypertrophy of smooth muscle cells? Hypertension 1997;29(3):770-775. doi:10.1161/01. hyp.29.3.770

10. Risler N, Castro C, Cruzado M, González S, Miatello R. Proteoglycans production by aortic vascular smooth muscle cells from hypertensive rats. Biocell 2003; 27(2):189196. doi:10.1161/01.hyp.34.4.893

11. Schwartz SM, Stemerman MB, Benditt EP: The aortic intima. II. Repair of the aortic lining after mechanical denudation. Am J Pathol 1975;81(1):15-42. PMID: 1180329

12. Yamin R, Morgan KG. Deciphering actin cytoskeletal function in the contractile vascular smooth muscle cell. Physiol 2012;590(17):4145-4154. doi: 10.1113/jphysiol.2012.232306

13. Guyton JR, Lindsay KL, Dao DT. Comparison of aortic intima and inner media in young adult versus aging rats. Stereology in a polarized system. Am J Pathol 1983; 111(2):234-246. PMID: 6846504
14. Ikari Y, McManus BM, Kenyon J, Schwartz SM. Neonatal Intima Formation in the Human Coronary Artery. Arterioscler Thromb Vasc Biol 1999; 19:2036-2040. doi:10.1161/01.atv.19.9.2036

15. Russell L, Holman RL, McGill HC Jr, Strong JP, Geer JC. The Natural History of Atherosclerosis. Am J Pathol 1958;34(2):209-235. PMCID: PMC1934740

16. Nakashima Y, Fujii H, Sumiyoshi S, Wight TN, Sueishi K. Early human atherosclerosis. Accumulation of lipid and proteoglycans in intimal thickenings followed by macrophage infiltration. Arterioscler Thromb Vasc Biol 2007;27:1159-1165. doi:10.1161/ATVBAHA.106.134080

17. Ross R: The pathogenesis of atherosclerosis: a perspective for the 1990s. Nature 1993;362(6423):801-809. DOI:10.1038/362801a0

18. Raines EW, Ross R: Smooth muscle cells and the pathogenesis of the lesions of atherosclerosis. Br Heart $J$ 1993;68(1 suppl):S30-S37. doi: 10.1136/hrt.69.1_suppl. s30

19. Kwon HM, Kang S, Hong BK, Kim D, Park HY, Shin MS, Byun KH. Ultrastructure changes of the external elastic lamina in experimental hypercholesterolemic porcine coronary arteries. Yonsei Med J 1999;40(3):273-282. doi:10.3349/ymj.1999.40.3.273

20. Wolinsky H. Long-term effects of hypertension on the rat aortic wall and their relation to concurrent aging changes. Morphological and chemical studies. Circ Res 1972;30(3):301-309. doi:10.1161/01.res.30.3.301

21. Arribas SM, Briones AM, Bellingham C, Gonzalez MC, Salaices M, Liu K, et al. Heightened aberrant deposition of hard-wearing elastin in conduit arteries of prehypertensive SHR is associated with increased stiffness and inward remodeling. Am J Physiol Heart Circ Physiol 2008;295(6):H2299-H2307. doi: doi.org/10.1152/ajpheart.00155.2008

22. McGrath JC, Deighan C, Briones AM, Shafaroudi MM, McBride M, Adler J, et al. New aspects of vascular remodeling: the involvement of all vascular cell types. Exp Physiol 2005;90(4):469-475. doi:10.1113/expphysiol.2005.030130

23. Lassègue $B$ \& Clempus RE. Vascular NAD(P)H oxidases: specific features, expression, and regulation. Am J Physiol Regul Integr Comp Physiol. 2003;285: R277-R297. doi:10.1152/ajpregu.00758.2002

24. Fortuño A, San José G, Moreno MU, Díez J \& Zalba G. Oxidative stress and vascular remodeling. Exp Physiol 2005;90:457-462. doi:10.1113/expphysiol.2005.030098 
25. Touyz RM. Intracellular mechanisms involved in vascular remodeling of resistance arteries in hypertension: role of angiotensin II. Exp Physiol. 2005;90:449-455. doi:10.1113/expphysiol. 2005.030080

26. Britton KA, Fox CS. Perivascular adipose tissue and vascular disease. Clin Lipidol. 2011;6(1):79-91. doi: 10.2217/ clp.10.89

27. Arribas SM, Hillier C, González C, McGrory S, Dominiczak AF, McGrath JC. Cellular aspects of vascular remodeling in hypertension revealed by confocal microscopy. Hypertension 1997;30(6):1455-1464. doi:10.1161/01. hyp.30.6.1455

28. Hayden MR, Karuparthi PR, Habibi J, Wasekar C, Lastra G, Manrique C, Stas S, Sowers JR. Ultrastructural islet study of early fibrosis in the Ren2 rat model of hypertension. Emerging role of the islet pancreatic pericyte-stellate cell. JOP 2007; 8(6):725-738. PMID:17993725

29. Hayden MR, Karuparthi PR, Habibi J, Lastra GL, Patel $\mathrm{K}$, Wasekar C, et al. Ultrastructure of islet microcirculation, pericytes and the islet exocrine interface in the HIP rat model of diabetes. Exp Biol Med (Maywood). 2008;233(9):1109-1123. doi: 10.3181/0709-RM-251

30. Hayden MR, Patel K, Habibi J, Gupta D, Tekwani SS, Whaley-Connell A, Sowers JR. Attenuation of endocrine-exocrine pancreatic communication in type 2 diabetes: pancreatic extracellular matrix ultrastructural abnormalities. J Cardiometab Syndr 2008;3(4):234-243. doi:10.1111/j.1559-4572.2008.00024.x

31. da Silva Meirelles L, Chagastelles PC, Nardi NB: Mesenchymal stem cells reside in virtually all post-natal organs and tissues. J Cell Sci 2006;119(Pt 11):2204-2213. doi: 10.1242/jcs.02932

32. Dore-Duffy P, Katychev A, Wang X, Van Buren E. CNS microvascular pericytes exhibit multipotential stem cell activity. J Cereb Blood Flow Metab 2006;26(5):613-624. doi:10.1038/sj.jcbfm.9600272

33. Armulik A, Abramsson A, Betsholtz C. Endothelial/pericyte interactions. Circ Res 2005;97(6):512-523. doi:10.1161/01.RES.0000182903.16652.d7

34. Allt G, Lawrenson JG. Pericytes: cell biology and pathology. Cells Tissues Organs. 2001;169(1):1-11. doi:10.1159/000047855

35. Sims DE. The pericyte. A review. Tissue Cell 1986;18:153174. doi:10.1016/0040-8166(86)90026-1

36. Hayden MR, Sowers JR. Isletopathy in Type 2 diabetes mellitus: implications of islet RAS, islet fibrosis, islet amyloid, remodeling, and oxidative stress. Antioxid Redox
Signal 2007;9(7):891-910. doi: 10.1089/ars.2007.1610

37. Andreeva ER, Pugach IM, Gordon D, Orekhov AN. Continuous subendothelial network formed by pericyte-like cells in human vascular bed. Tissue Cell 1998; 30(1):127135. https://doi.org/10.1016/S0040-8166(98)80014-1

38. Mitchell GF, Hwang SJ, Vasan RS et al. Arterial stiffness and cardiovascular events: the Framingham Heart Study. Circulation 2010; 121(4): 505-511. doi: 10.1161/ CIRCULATIONAHA.109.886655.

39. DeMarco VG, Habibi J, Jia G et al. Low-Dose mineralocorticoid receptor blockade prevents Western dietinduced arterial stiffening in female mice. Hypertension 2015;66(1):99-107. doi: 10.1161/HYPERTENSIONAHA.115.05674

40. Weisbrod RM, Shiang T, Al Sayah L, Fry JL, Bajpai $\mathrm{S}$, Reinhart-King CA, et al. Arterial stiffening precedes systolic hypertension in diet-induced obesity. Hypertension 2013;62(6):1105-1110. doi:10.1161/HYPERTENSIONAHA.113.01744.

41. Wolinsky H, Glagov S. Comparison of abdominal and thoracic aortic medial structure in mammals. Circ Res 1969;25(6):677-686. doi:10.1161/01.res.25.6.677

42. Dingemans KP, Teeling P, Lagendijk JH, Becker AE. Extracellular matrix of human aortic media: An ultrastructural histochemical and immunohistochemical study of human aortic media. Anat Rec 2000;258(1):1-14. doi:10.1002/(SICI)1097-0185(20000101)258:1<1::AIDAR1>3.0.CO;2-7

43. Wolinsky H, Glagov S. A Lamellar Unit of Aortic Medial Structure and Function in Mammals. Circ Res 1967;20(1):99-111. DOI:10.1161/01.RES.20.1.99

44. Chaldakov GN, Vankov VN. Morphological aspects of secretion in the arterial smooth muscle cell, with special reference to the Golgi complex and microtubular cytoskeleton. Atherosclerosis 1986; 61: 175-192.

45. Chaldakov GN. GEORGE E. PALADE LECTURE. Human body as a multicrine system, with special reference to cell protein secretion: From vascular smooth muscles to adipose tissue. Biomed Rev 2016; 27: VIII-XIX

46. Majesky MW, Dong XR, Hoglund V, Mahoney WM Jr, Daum G. The adventitia: a dynamic interface containing resident progenitor cells. Arterioscler Thromb Vasc Biol 2011;31(7):1530-9. doi: 10.1161/ATVBAHA.110.221549.

47. Majesky MW, Dong XR, Hoglund V, Daum G, Mahoney WM Jr. The adventitia: a progenitor cell niche for the vessel wall. Cells Tissues Organs 2012;195(1-2):73-81. doi: 10.1159/000331413. 